\title{
Pleomorphic mesothelioma: report of 10 cases
}

\author{
Nelson G Ordóñez
}

Department of Pathology, The University of Texas MD Anderson Cancer Center, Houston, TX, USA

\begin{abstract}
Mesotheliomas with pleomorphic features are rare and only a few studies on this mesothelioma variant have been published. Little information regarding the immunoprofile of these tumors and none on their electron microscopic features was included in these studies. Herein are reported 10 cases of pleomorphic mesothelioma that were investigated using a large panel of immunohistochemical markers, 4 of which were also studied by electron microscopy. All of the patients were men and seven had a history of asbestos exposure. Nine of the cases originated in the pleura and one in the peritoneum. Histologically, the tumors were characterized by being composed of large, often discohesive, cells that varied in size and shape, had dense abundant eosinophilic cytoplasm, and single or multiple irregular nuclei, which often contained one or several large nucleoli. Mitotic activity was high and atypical mitoses frequent. Immunoreactivity for pan-keratin and keratin 7 was strong in all of the cases. Expression for calretinin, WT1, podoplanin, mesothelin and keratin 5/6 was also frequent, but variable. All cases were negative for MOC-31, carcinoembryonic antigen, CD15, TAG-72 and thyroid transcription factor-1. Electron microscopy often showed the presence of abundant long, slender microvilli on the cell membrane of the neoplastic cells. These findings demonstrate that, contrary to what has been suggested by some investigators, both immunohistochemistry and electron microscopy can be very helpful in assisting in the diagnosis of pleomorphic mesotheliomas. That the seven patients who underwent extrapleural pneumonectomy had extensive lymph node metastasis and that the median survival of those patients for whom follow-up information was available was only $\mathbf{8 . 2}$ months indicates that mesotheliomas with pleomorphic features are associated with highly aggressive clinical behavior. Therefore, when this subtype of epithelioid mesothelioma is present, it should be reported as it can significantly affect the prognosis and treatment of the patient. Modern Pathology (2012) 25, 1011-1022; doi:10.1038/modpathol.2012.39; published online 2 March 2012
\end{abstract}

Keywords: electron microscopy; histopathology; immunohistochemistry; mesothelioma; pleomorphic

A well-known characteristic of mesotheliomas is their ability to grow in a wide variety of histologic patterns. Based on their light microscopic features, the 2004 WHO Classification defines four major histologic subtypes of diffuse malignant mesothelioma: epithelioid, sarcomatoid, mixed epithelioid and sarcomatoid (biphasic), and desmoplastic. ${ }^{1}$ Although epithelioid mesotheliomas most frequently present a tubulopapillary, adenomatoid (microglandular) or solid pattern, on rare occasions, they may present other histologic patterns, including deciduoid, ${ }^{2-4}$ clear cell, ${ }^{5,6}$ adenoid cystic, ${ }^{7}$ pleomorphic,${ }^{8,9}$ small cell, ${ }^{10,11}$ rhabdoid,${ }^{12}$ glomeruloid, ${ }^{13}$ signet-ring cell ${ }^{14,15}$ and oncocytoid. ${ }^{16}$ Some of these patterns, such as the pleomorphic, however, have not been well defined. This is apparent in a recent publication in which it was suggested that

Correspondence: Dr NG Ordóñez, MD, Department of Pathology, The University of Texas MD Anderson Cancer Center, 1515 Holcombe Boulevard, Houston, TX 77030, USA.

E-mail: nordonez@mdanderson.org

Received 12 December 2011; revised 4 January 2012; accepted 4 January 2012; published online 2 March 2012 pleomorphic mesothelioma should be regarded as a sarcomatoid pattern, rather than a subtype of diffuse epithelioid malignant mesothelioma as it is currently considered in the WHO Classification. ${ }^{9}$ In a review of the literature, I was able to find only three publications that focused primarily on pleomorphic mesothelioma, ${ }^{8,9,17}$ one of which was a case report. To my knowledge, with the exception of that single case report, no other study has been published in which the immunophenotype of a pleomorphic mesothelioma was investigated. The lack of sufficient information on the immunoprofile of pleomorphic mesothelioma and the need to better define the criteria for the diagnosis of this histologic variant of mesothelioma prompted the investigation of 10 such cases by immunohistochemistry. Electron microscopy was also performed in four of these cases. Nine cases originated in the pleura and one in the peritoneum. At present, no study on the ultrastructure of pleomorphic mesothelioma has been published. Additionally, peritoneal pleomorphic epithelioid mesotheliomas have not been previously documented. 


\section{Materials and methods}

Ten cases of pleomorphic mesothelioma were identified from a review of 640 cases of mesothelioma from the files of the Department of Pathology at The University of Texas MD Anderson Cancer Center, Houston, TX, USA. Nine patients were referred to this institution for further evaluation and treatment, and one case was sent in consultation to the author. Hematoxylin-and-eosin-stained sections were available in all cases. In addition, cytology preparations of a pleural effusion were available in one case. Immunohistochemical studies were performed on formalin-fixed, paraffin-embedded tissue specimens using the avidin-biotinperoxidase complex method in a Dako AutoStainer (Carpinteria, CA, USA). The primary antibodies used are listed in Table 1. The immunostaining was carried out using the LSAB2 peroxidase kit (Dako). To enhance the immunostaining, a heat epitope retrieval procedure was performed using a Black-and-Decker vegetable steamer (Shelton, CT, USA) as previously described. ${ }^{18}$ The antigen-antibody immunoreaction was visualized using either 3amino-9-ethylcarbazole or 3,3'-diaminobenzidine as chromogen. To evaluate the specificity of the immunoreaction, known positive and negative tissues were used as controls. The immunostaining was graded on a sliding scale of $1+$ to $4+$ according to the percentage of reactive cells (trace, $<1 \% ; 1+$, $1-25 \%$; $2+, 26-50 \%$; $3+, 51-75 \% ; 4+,>75 \%$ ). For ultrastructural studies, samples of the tumor were fixed in $2 \%$ glutaraldehyde in phosphate buffer, post-fixed in $1 \%$ osmium tetroxide and embedded in epon epoxy resin. Ultrathin sections were stained with uranyl acetate and lead citrate.

\section{Results}

\section{Clinical Findings}

All 10 patients were men, ranging in age of 61 to 74 years (mean, 66. 7). There was a history of asbestos exposure in seven patients and smoking in five.
In nine of the cases, the mesothelioma originated in the pleura and in one, in the peritoneum. Two of the patients received chemotherapy alone; seven underwent extrapleural pneumonectomy with four of these also receiving radiotherapy after surgery and one chemotherapy before surgery. Treatment information was not available in the remaining patient. Six of the seven patients for whom follow-up information was available died of recurrent disease 3 to 7 months after diagnosis (mean, 7.3 months) and one is alive with recurrent disease 3 months after extrapleural pneumonectomy.

\section{Pathology Findings}

Gross examination of the seven extrapleural pneumonectomy specimens showed diffuse involvement of the visceral and parietal pleura with encasement of the lung. Three cases had involvement of the pericardium, diaphragm and adjacent lung parenchyma (cases 7, 8 and 10), and one each of the diaphragm and pericardium (case 2), diaphragm and lung (case 6), pericardium alone (case 9) and adjacent lung parenchyma alone (case 4). Multiple lymph nodes were involved by metastasis in all of the cases. The most significant light microscopic findings are summarized in Table 2. Histologically, the tumor present in the biopsy material was entirely composed of pleomorphic epithelioid cells (cases 1, 3 and 5); whereas, the proportion of the pleomorphic cell component ranged from 20 to $100 \%$ in the pneumonectomy specimens (cases 2,4 , $6,7,8,9$ and 10). In all of the cases, the tumor usually appeared to be made of large discohesive epithelioid cells that varied in size and shape, and contained abundant, dense eosinophilic cytoplasm (Figures 1a, b, 2a, b and 3a-c). The nuclei were irregular and either single or multiple, and the chromatin often clumped and irregularly distributed throughout the nucleus (Figures $1 \mathrm{~b}, 2 \mathrm{~b}$ and $3 \mathrm{~d}$ ). Large single or multiple nucleoli were often seen (Figures $2 \mathrm{~b}$ and $3 \mathrm{~b}, \mathrm{~g}$ ). In two cases (cases 4 and 5), there were focal areas in which the cells contained intracytoplasmic vacuoles (Figure 2c). Although in

Table 1 Antibodies used in this study

\begin{tabular}{|c|c|c|c|c|}
\hline Marker & Source & Tуре & Dilution & Antigen retrieval \\
\hline Calretinin & Zymed (South San Francisco, CA, USA) & PAb (rabbit) & $1: 20$ & Yes (citrate) \\
\hline Pan-keratin & Dako (Carpinteria, CA, USA) & AE1/AE3 MAbs & $1: 50$ & Yes (citrate) \\
\hline Keratin 7 & Dako & OV-TL 12/30 & $1: 100$ & Yes (enzymatic digestion) \\
\hline Keratin 5/6 & Dako & D5/16B4 MAb & $1: 100$ & Yes (citrate) \\
\hline WT1 & Dako & 6F-H2 MAb & $1: 40$ & Yes (Tris-EDTA) \\
\hline Podoplanin & Signet Laboratories (Dedham, MA, USA) & $\mathrm{D} 2-40 \mathrm{MAb}$ & $1: 25$ & Yes (Tris-EDTA) \\
\hline Mesothelin & Novocastra (Newcastle-upon-Tyne, UK) & $5 \mathrm{~B} 2$ & $1: 30$ & Yes (Tris-EDTA) \\
\hline MOC-31 & Dako & MAb & $1: 50$ & Yes (citrate) \\
\hline CEA & Lab Vision (Fremont, CA, USA) & PAb (rabbit) & $1: 200$ & No \\
\hline CD15 & Becton-Dickinson (Mountainview, CA, USA) & Leu-M1 MÁb & $1: 40$ & Yes (Tris-EDTA) \\
\hline TAG-72 & BioGenex (San Ramon, CA, USA) & B72.3 MAb & $1: 300$ & No \\
\hline TTF-1 & Dako & 8G7G3/1 MAb & $1: 25$ & Yes (citrate) \\
\hline
\end{tabular}


Table 2 Summary of clinical and pathologic findings

\begin{tabular}{|c|c|c|c|c|c|c|}
\hline Case & Sex/age & Type of specimen & Location & Light microscopy (\% pleomorphic cell component) & Treatment & Follow-up (months) \\
\hline 1 & $\mathrm{M} / 72$ & Biopsy & Rt. pleura & $\begin{array}{l}\text { Mixture of discohesive, often multi-nucleated, cells that } \\
\text { exhibit a great variation in their size and shape }(100 \%) \text {. } \\
\text { Sometimes the pleomorphic cells appeared to be lining } \\
\text { irregular spaces or poorly formed papillae. Numerous, } \\
\text { often atypical, mitoses were present. }\end{array}$ & Chemotherapy & INA \\
\hline 2 & $\mathrm{M} / 71$ & Pneumonectomy & Lt. pleura & $\begin{array}{l}\text { About } 25 \% \text { of the tumor was composed of pleomorphic } \\
\text { epithelioid cells that were often multinucleated. }\end{array}$ & Pneumonectomy+radiotherapy & DOD (4) \\
\hline 3 & $\mathrm{M} / 56$ & Biopsy & Peritoneum & $\begin{array}{l}\text { Diffuse proliferation of pleomorphic epithelioid cells, } \\
\text { some of which contained multiple nuclei }(100 \%) \text {. } \\
\text { Numerous mitoses, many atypical, were seen. }\end{array}$ & INA & INA \\
\hline 4 & $\mathrm{M} / 74$ & Pneumonectomy & Lt. pleura & $\begin{array}{l}\text { About } 80 \% \text { of the tumor composed of large, often } \\
\text { multinucleated, epithelioid cells arranged in irregular, } \\
\text { solid nests. High mitotic activity, prominent lymphatic } \\
\text { invasion and extensive necrosis. }\end{array}$ & Pneumonectomy+radiotherapy & DOD (17) \\
\hline 5 & $\mathrm{M} / 62$ & Biopsy & Rt. pleura & $\begin{array}{l}\text { Diffuse proliferation of discohesive cells with abundant } \\
\text { cytoplasm, many of which were multinucleated (100\%). } \\
\text { Mitoses, including atypical mitoses, were numerous. }\end{array}$ & Chemotherapy & INA \\
\hline 6 & $\mathrm{M} / 67$ & Pneumonectomy & Lt. pleura & $\begin{array}{l}\text { About } 30 \% \text { of the tumor was composed of a diffuse } \\
\text { proliferation of pleomorphic epithelioid cells, some of } \\
\text { which were multinucleated. Numerous mitoses, } \\
\text { including some that were atypical, were present. }\end{array}$ & Pneumonectomy & DOD (3) \\
\hline 7 & $\mathrm{M} / 64$ & Pneumonectomy & Rt. pleura & $\begin{array}{l}\text { About } 25 \% \text { of the tumor was composed of pleomorphic } \\
\text { epithelioid cells that were often multinucleated. }\end{array}$ & Pneumonectomy+radiotherapy & DOD (4) \\
\hline 8 & $\mathrm{M} / 73$ & Pneumonectomy & Rt. pleura & $\begin{array}{l}\text { About } 15 \% \text { of the tumor was composed of pleomorphic } \\
\text { epithelioid cells, some of which were multinucleated. }\end{array}$ & Chemotherapy+pneumonectomy & DOD (6) \\
\hline 9 & $\mathrm{M} / 67$ & Pneumonectomy & Lt. pleura & $\begin{array}{l}\text { About } 20 \% \text { of the tumor was composed of pleomorphic } \\
\text { epithelioid cells, some of which were multinucleated. }\end{array}$ & Pneumonectomy+radiotherapy & DOD (10) \\
\hline 10 & $\mathrm{M} / 61$ & Pneumonectomy & Rt. pleura & $\begin{array}{l}\text { Diffuse proliferation of pleomorphic epithelioid cells, } \\
\text { many of which were multi-nucleated }(100 \%) \text {. On } \\
\text { occasion, the pleomorphic cells appeared to be lining } \\
\text { poorly formed tubular-like structures. Prominent } \\
\text { lymphatic invasion present. }\end{array}$ & Pneumonectomy & AWD (3) \\
\hline
\end{tabular}

Abbreviations: AWD, alive with disease; DOD, died of disease; INA, information not available. 

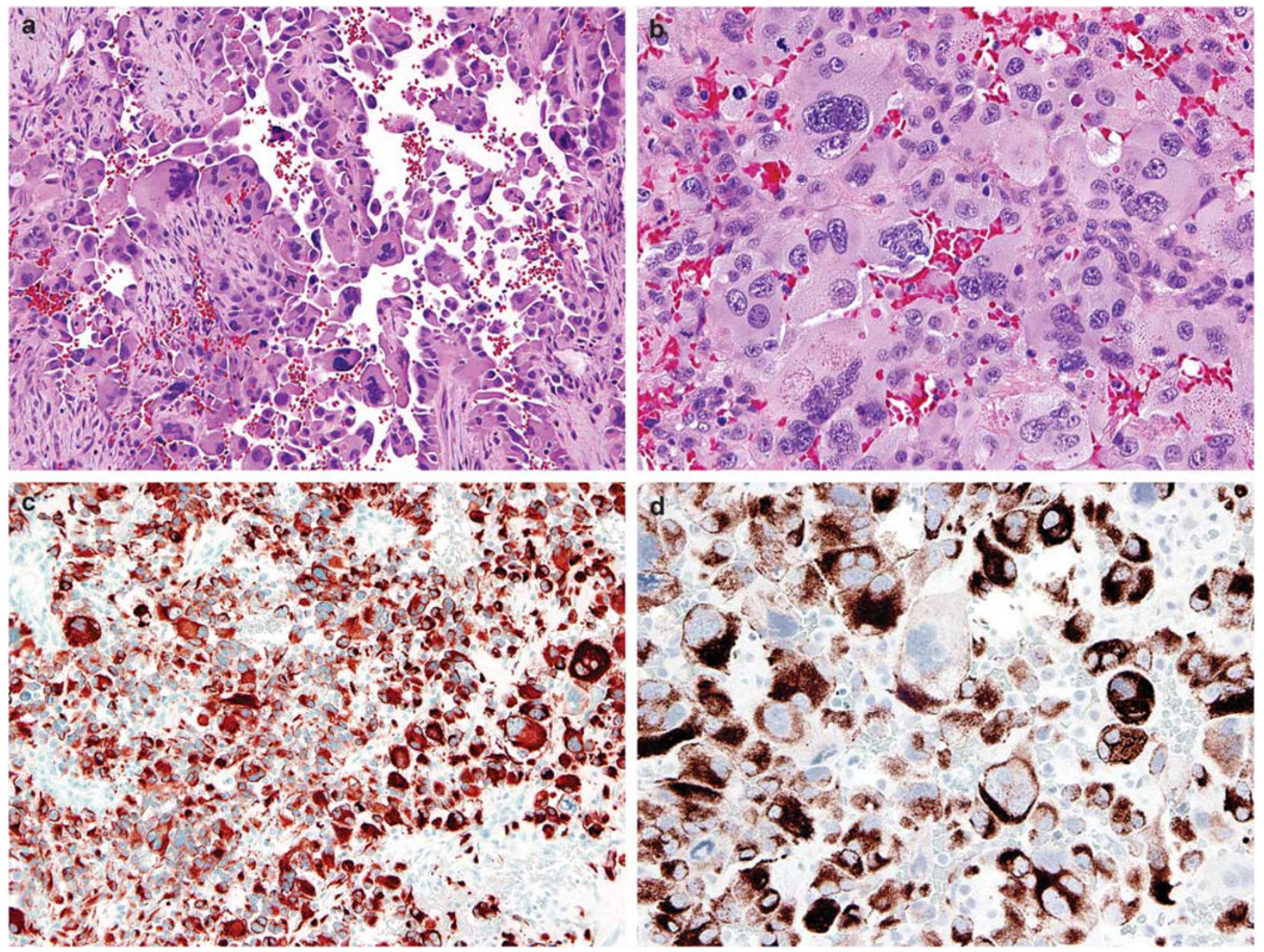

Figure 1 Case 1: (a) The tumor cells appeared to be lining an irregular space and a papillae-like structure (center). There is marked pleomorphism of the neoplastic cells, some of which are multinucleated. Two mitotic figures can be seen in the center of the figure. (b) Higher magnification of the neoplastic cells, many of which are multinucleated. The cytoplasm is abundant and the nuclei often exhibit clumped chromatin. Two mitoses are present in the upper left corner of the figure. (c) Immunohistochemical preparations showing diffuse strong positivity for pan-keratin in the cytoplasm of the neoplastic cells. (d) Strong keratin expression can be seen in both mononucleated and multinucleated tumor cells.

most of the cases the tumor consisted of a diffuse proliferation of pleomorphic epithelioid cells with a variable number of multinucleated cells (Figures 3a and f), in one (case 4), the neoplastic cells were arranged in solid nests often exhibiting central necrosis (Figure 2a). In some areas of the tumor in one case (case 1), the neoplastic cells appeared to be lining irregular spaces or forming papillary-like structures (Figure 1a); whereas, in another (case 10), the cells appeared to be arranged in a tubularlike array (Figure 3h). An interesting finding in one of the cases (case 5) was that the mesothelioma, in addition to presenting pleomorphic morphology, also had areas resembling deciduoid mesothelioma, but which were associated with numerous multinucleated cells and a large number of mitoses that were often atypical (Figures 3a and b). In all of the cases, the mitotic activity was high (over 20 per 10 high-power fields) and atypical mitoses were frequently seen (Figure 3b). Two of the cases (cases 4 and 10) exhibited prominent lymphatic invasion (Figures 2d and e). In the one case (case 10) for which cytology preparations of the pleural effusion were available for review, numerous tumor cells exhibiting features of pleomorphic mesothelioma were seen (Figure 4).

\section{Immunohistochemistry}

Immunohistochemical results are summarized in Table 3. The pleomorphic cells in all 10 cases strongly reacted for pan-keratin and keratin 7 . They were also positive for keratin $5 / 6$ in six of the eight cases investigated, but the staining tended to be more focal than that for keratin 7 . The staining for all of the other positive mesothelioma markers investigated, including calretinin, WT1, mesothelin and podoplanin, was variable. In all, 8 of 10 cases were positive for calretinin, 4 of 6 for WT1, 6 of 7 for 
mesothelin and 5 of 7 for podoplanin. The immunoreactivity was both nuclear and cytoplasmic for calretinin, and it was nuclear for WT1; whereas, it usually occurred along the cell membrane for both mesothelin and podoplanin. Strong positivity for podoplanin was also seen in the lymphatics, which not only served as a positive internal control, but also allowed the lymphatic invasion by tumor to be evaluated. None of the cases that were stained for MOC-31, CEA, CD15, TAG-72 or TTF-1 were positive for any of these markers.

\section{Electron Microscopic Findings}

The ultrastructural features of all four of the cases for which tissue was submitted for electron microscopic evaluation (cases 4, 6, 7 and 10) were very similar. The neoplastic cells often appeared discohesive and the cell junctions in the areas in which the cells remained attached to each other were often small and poorly developed (Figure 5a). The apical and lateral surfaces of the cell membrane were covered by a large number of long, slender microvilli (Figures 5a-c and $6 b)$. The nuclei were pleomorphic, single or multiple, often exhibited markedly irregular contours, and contained one or several large nucleoli (Figures 5ac). The cytoplasm was abundant and had a variable number mitochondria, short profiles of rough endoplasmic reticulum and abundant intermediate filaments that sometimes appeared to be arranged in bundles (Figure $5 \mathrm{~d}$ ). In one case (case 4), some of the neoplastic cells exhibited intracytoplasmic lumina, sometimes containing electron dense material, presumably hyaluronic acid or hyaluronic acidcontaining proteoglycans (Figure $5 \mathrm{~d}$ ). In another case (case 6), the cytoplasm of some cells contained a large number of tubules with a honeycomb structure
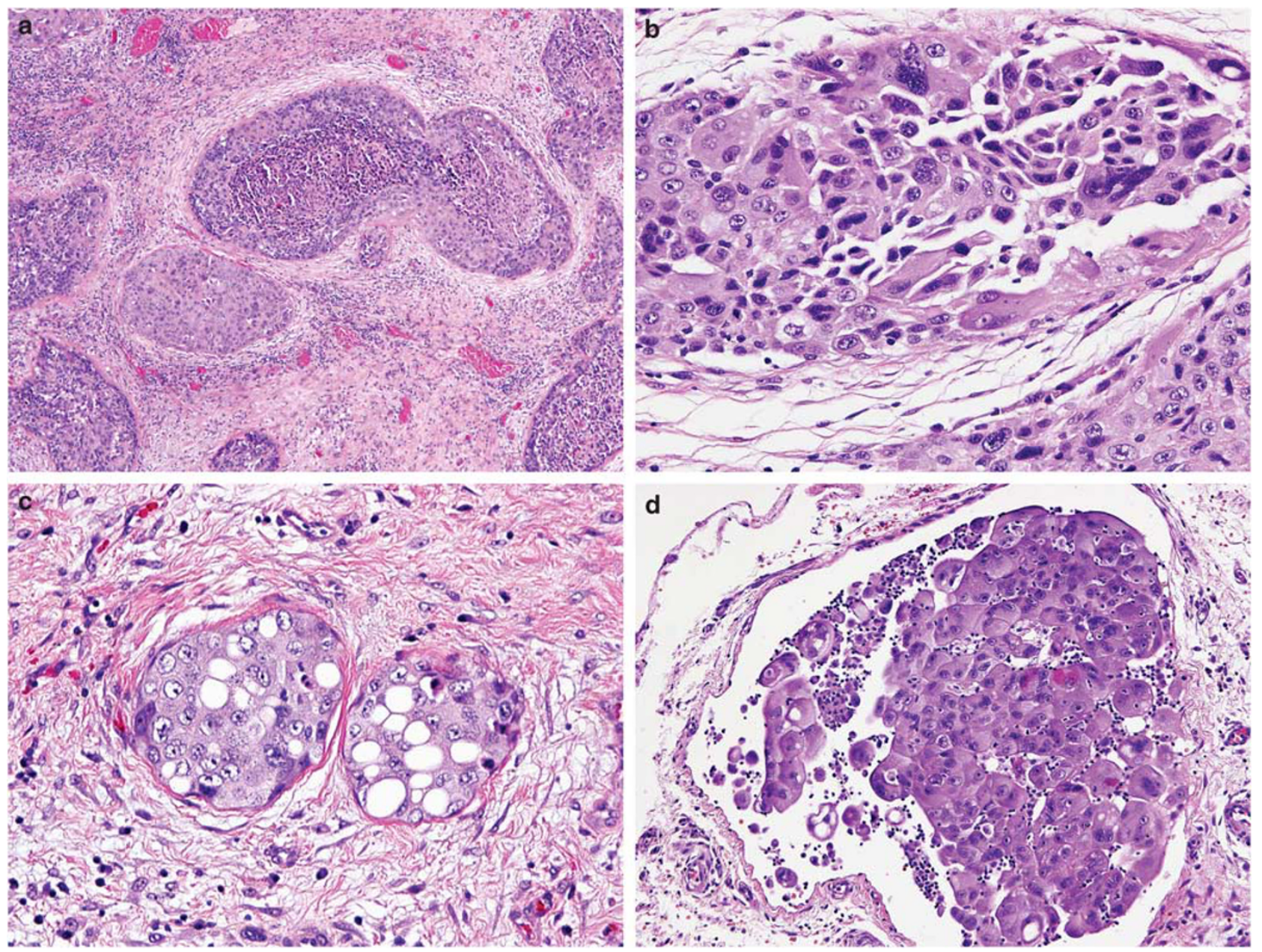

Figure 2 Case 4: (a) The tumor appears to be arranged in irregular solid nests of pleomorphic epithelioid cells. Some of the clusters exhibit prominent central necrosis. (b) Higher magnification of one of the nests showing that they are composed of neoplastic cells presenting a great variation in size in shape. (c) Two clusters of tumor cells, some of which have vacuolated cytoplasm most probably due to the presence of intracytoplasmic lumina. The surrounding stroma exhibits myxoid features. (d) Lymphatic vessel showing a lumen almost occluded by large pleomorphic tumor cells, some of which are multinucleated. (e) Immunostaining for podoplanin showing positivity along the lymphatic endothelium as well as in the cell membrane of the neoplastic cells. (f) Consecutive section showing nuclear and cytoplasmic positivity for calretinin. (g) Nuclear positivity for WT1 in some of the neoplastic cells. (h) Tumor cells exhibiting strong membranous reactivity for mesothelin. 

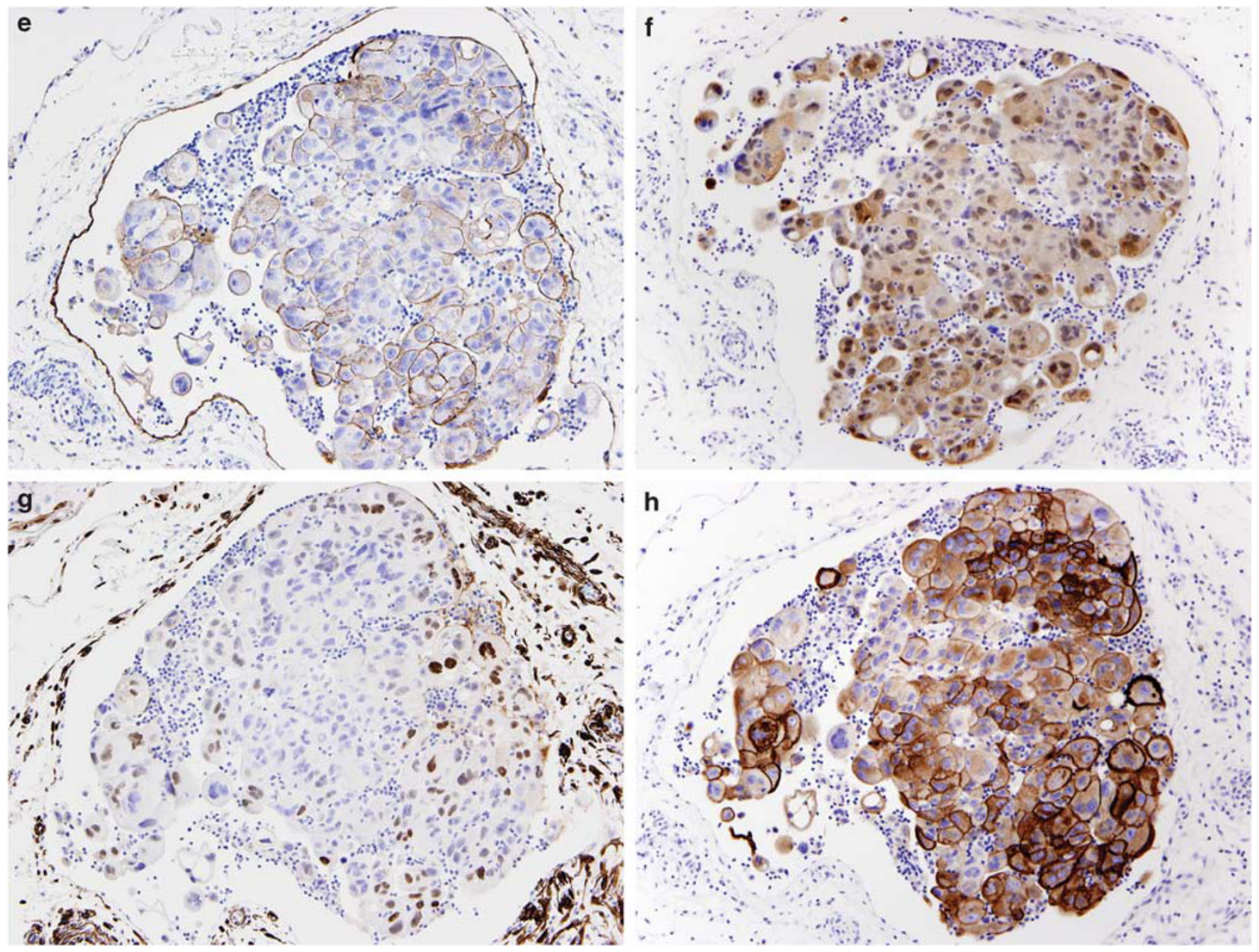

Figure 2 Continued.

located within the cisternae of the rough endoplasmic reticulum (Figures 6a-c).

\section{Discussion}

One of the main characteristics of tumors derived from the serosal membranes is the wide variation in their histologic appearance. Histologically, mesotheliomas have been classified into three major variants: epithelioid, spindle, and mixed or biphasic. Epithelioid mesotheliomas typically present a tubulopapillary or solid pattern, but, on occasion, they may exhibit adenomatoid, deciduoid, clear cell, signet-ring or small cell features. The term pleomorphic mesothelioma is used to designate a variant of epithelioid mesothelioma that is characterized by being composed of pleomorphic epithelioid cells showing nuclear enlargement, hyperchomasia, prominent nucleoli and, frequently, multinucleation, thus resembling a pleomorphic carcinoma. ${ }^{1,17,19}$ Although illustrations of tumors exhibiting such morphology are commonly provided in major books and review articles on the pathology of mesotheliomas, only a few studies have been published primarily focusing on this uncommon variant of mesothelioma. One of these studies was a case report by Itami et $a l^{8}$ on a case of pleomorphic mesothelioma with osteoclast-like cells; the second, a series of 44 cases by Galateau-Salle et $a{ }^{17}$ published only in abstract form; and the third and most recent by Kadota et $a l^{9}$ on 34 cases that they identified among 305 mesothelioma cases from the Sloan-Kettering Cancer Center. With the exception of the case report by Itami et al, in which the tumor was investigated by immunohistochemistry using a large panel of antibodies, little information exists regarding the immunoprofile of pleomorphic mesotheliomas. In the Kadota et al series, the authors mentioned, in the Materials and methods section, that the immunostaining for a variety of markers, including calretinin, WT1, keratin 5/6, D2-40, CEA, CD15, B72.3 and Ber-EP4, was done in order to establish the diagnosis of diffuse pleural mesothelioma; however, no results were provided specifically in regards to the expression of these markers in the pleomorphic component of the tumors. Additionally, in the 2006 AFIP fascicle on Pathology in Tumors of the Serosal Membranes, it was stated that pleomorphic mesotheliomas may not stain 
for any antigen, except broad-spectrum keratin, and may not have specific ultrastructural features. ${ }^{19}$

The results of this study show that, in general, pleomorphic mesotheliomas maintain the immunophenotype seen in other types of epithelioid mesotheliomas, particularly their expression of both pan-keratin and keratin 7 , which were found to be diffusely and strongly positive in all 10 cases in the pleomorphic mesothelioma component. Immunoreactivity for the other positive mesothelioma markers investigated (keratin 5/6, calretinin, WT1, mesothelin and podoplanin) was more variable, as none of these were found to be expressed in all of the cases and the staining was sometimes focal. Also, in contrast to the experience of other investigators, ${ }^{19}$ electron microscopy frequently demonstrated the presence of some of the ultrastructural features that are regarded as being characteristic of mesothelial differentiation, such as the presence of numerous long, slender microvilli on the apical and lateral surfaces of the cell membrane, in the pleomorphic neoplastic cell component. An interesting ultrastructural finding in one of the cases was the presence of tubular crystalloid inclusions within the cisternae of the endoplasmic reticulum. Crystalloids, such as those seen in this case, were first described by Henderson et $a l^{20}$ in the neoplastic cells of a pleural effusion from a patient with mesothelioma and were thought to be secretory products of the rough endoplasmic reticulum that could not be transported or secreted by the cell. Hammar et $a l^{21}$ reported four cases of mucicarmine positive mesothelioma in which tubular crystalloids were found to be associated with the microvilli in all
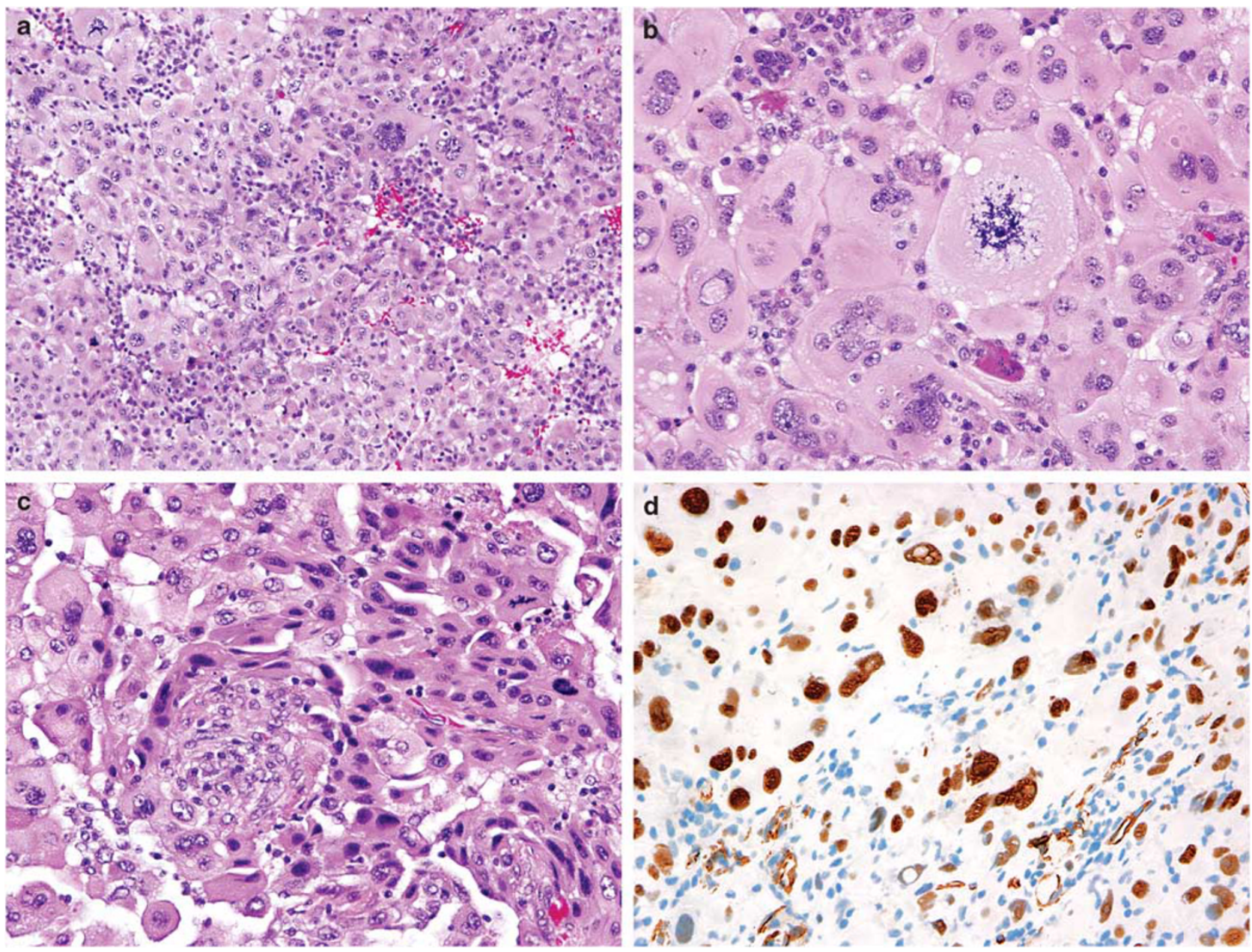

Figure 3 Case 5: (a) Diffuse proliferation of large epithelioid cells with abundant dense eosinophilic cytoplasm and deciduoid features. Many of the cells are multinucleated. An atypical mitosis can be seen near the center of the figure. (b) Higher magnification showing a detail of the morphology of the cells. An atypical mitosis is present in the center of the figure. (c) Area of the same tumor in which the cells appear more pleomorphic and some of them are elongated. Two mitotic figures are present toward the right upper corner. (d) The nuclei of the neoplastic cells are markedly pleomorphic and strongly positive for WT1. (e) Tumor cells strongly reacting for mesothelin along the cell membrane. Case 10: (f) Pleomorphic epithelioid cells diffusely infiltrating the skeletal muscle of the chest wall. (g) Higher magnification showing, in more detail, the morphologic features of the neoplastic cells which exhibit single or multiple nuclei and often contain a large nucleolus. Note that the cell cytoplasm is less abundant than that seen in case 5. A tripolar mitosis is seen in the lower left corner of the figure. (h) An area of the same tumor in which the neoplastic cells appear to be discohesive and are sometimes arranged in a poorly formed tubular array. 

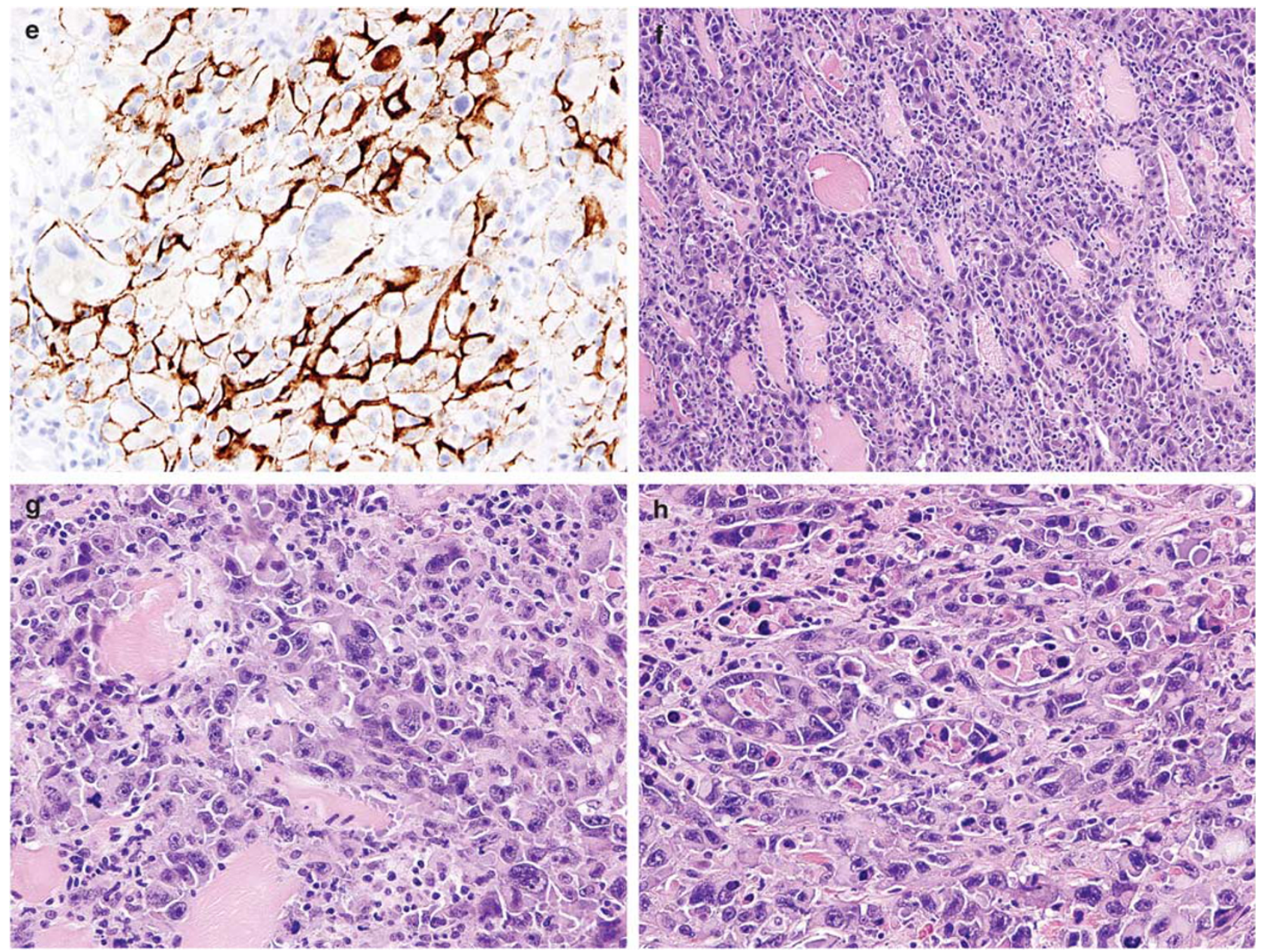

Figure 3 Continued.

four cases, as well as in the cytoplasm in one. More recently, in a prospective ultrastructural study of 59 epithelioid mesotheliomas, crystalloid inclusions were found in $9(15 \%)$ of the cases, which indicates that crystalloids are not as rare a finding in mesotheliomas as was previously thought. ${ }^{16}$ This study also concluded that, when present, these crystalloids can serve as a helpful ultrastructural marker for assisting in the diagnosis of mesotheliomas because of their unique morphology.

Mesotheliomas with pleomorphic features can be confused with a variety of other tumors with similar morphology that can involve the serosal membranes. The tumor with the greatest potential of being confused with mesotheliomas with pleomorphic features are pleomorphic carcinomas of the lung. In these instances, immunohistochemical studies can greatly facilitate the differential diagnosis. In my experience, pleomorphic carcinomas of the lung, in contrast to pleomorphic mesotheliomas, do not express calretinin, mesothelin or WT1 and, therefore, immunostaining for these markers can assist in discriminating between these two malignancies. TTF-1, which is invariably absent in mesotheliomas, has been reported to be expressed

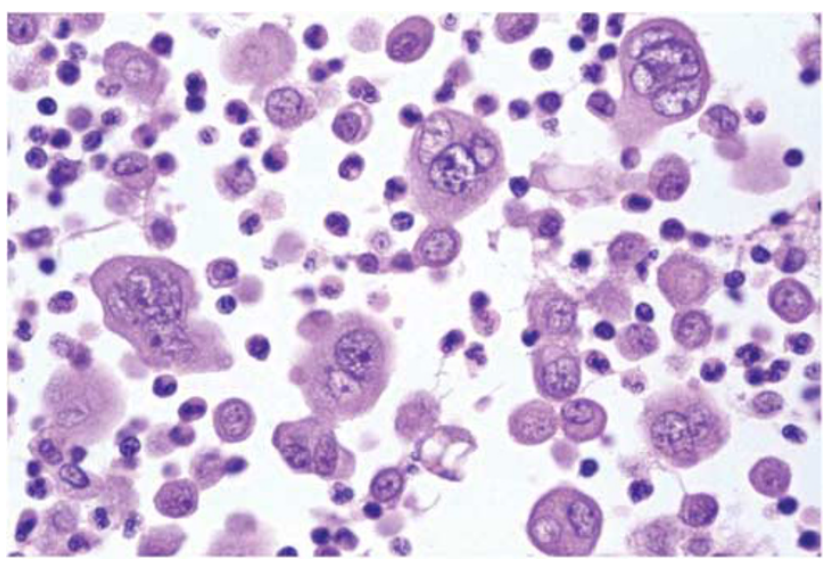

Figure 4 Case 10. Cytology preparation of pleural effusion showing several epithelioid tumor cells with single or multiple, irregular nuclei exhibiting features similar to those in the neoplastic cells of Figure 3g.

in about $50 \%$ of the cases of pleomorphic carcinoma of the lung; ${ }^{22}$ therefore, when immunostaining for this marker is present, it may help to establish the lung origin of a pleomorphic carcinoma involving the pleura. 
Table 3 Immunohistochemical results

\begin{tabular}{lcccccccccc}
\hline & Case 1 & Case 2 & Case 3 & Case 4 & Case 5 & Case 6 & Case 7 & Case 8 & Case 9 & Case 10 \\
\hline Calretinin & 0 & $2+$ & $2+$ & $4+$ & $3+$ & $3+$ & $2+$ & $3+$ & $4+$ \\
Pan-keratin & $4+$ & $4+$ & $4+$ & $4+$ & $4+$ & $4+$ & $4+$ & $4+$ & $4+$ & $4+$ \\
Keratin 7 & $4+$ & $4+$ & $4+$ & $4+$ & $4+$ & $4+$ & $4+$ & $4+$ & $4+$ & $4+$ \\
Keratin 5/6 & 0 & $2+$ & ND & $3+$ & $3+$ & $2+$ & ND & $1+$ & $3+$ & 0 \\
WT1 & 0 & ND & $4+$ & $3+$ & ND & $3+$ & ND & $1+$ & ND & 0 \\
Podoplanin & 0 & $2+$ & ND & $3+$ & ND & $3+$ & ND & 0 & $4+$ & $1+$ \\
Mesothelin & $1+$ & ND & $4+$ & $4+$ & ND & $4+$ & ND & 0 & $4+$ & $2+$ \\
MOC-31 & 0 & 0 & 0 & 0 & 0 & 0 & 0 & 0 & 0 \\
CEA & 0 & 0 & 0 & 0 & 0 & 0 & ND & 0 & 0 \\
CD15 & 0 & 0 & 0 & 0 & 0 & 0 & 0 & 0 & 0 \\
TAG-72 & 0 & 0 & 0 & 0 & 0 & 0 & 0 & 0 & 0 \\
TTF-1 & 0 & 0 & 0 & 0 & 0 & 0 & 0 & 0 & 0 \\
\hline
\end{tabular}

Abbreviations: CEA, carcinoembryonic antigen; ND, not done; TTF-1, thyroid transcription factor-1.

Another type of carcinoma with which pleomorphic mesotheliomas can potentially be confused is anaplastic thyroid carcinoma, as these tumors frequently metastasize to the lung and may involve the pleura. As in the case of pleomorphic carcinomas of the lung, anaplastic thyroid carcinomas do not express calretinin, mesothelin or WT1, but can express Pax8, ${ }^{23,24}$ a marker that is absent in mesotheliomas. ${ }^{25}$ TTF-1 has little value as it has been reported to be expressed only in a small percentage of anaplastic thyroid carcinomas. ${ }^{23,26}$

Pleomorphic mesotheliomas can also be confused with some sarcomas. The two sarcomas with the greatest potential of being confused with pleomorphic mesotheliomas are angiosarcomas and the pleomorphic variant of malignant fibrous histiocytoma. Pleural angiosarcomas are often epithelioid and can easily be mistaken for a mesothelioma, both clinically and histologically, ${ }^{27-30}$ and examples of epithelioid pleural angiosarcomas exhibiting marked pleomorphism that could easily be confused with pleomorphic mesotheliomas have been documented in the literature. ${ }^{31}$ The pleural biopsy of one of the mesotheliomas cases in this study (case 1), in addition to being somewhat hemorrhagic, showed areas in which the neoplastic cells appeared to be lining irregular spaces resembling the vascular spaces seen in an angiosarcoma (Figure 1a). As a result of this, the possibility of an angiosarcoma was considered in the differential diagnosis; however, the strong keratin expression and the mesothelin positivity, together with the lack of reactivity for the endothelial markers CD31 and CD34, allowed the diagnosis of angiosarcoma to be ruled out.

As pleomorphic malignant fibrous histiocytomas can arise in the chest wall and involve the pleura, they can potentially be confused with pleomorphic mesotheliomas, especially in small biopsies. Similar to pleomorphic mesotheliomas, pleomorphic malignant fibrous histiocytomas have a large population of multinucleated malignant cells, but in contrast to those tumors, they also have a large fibroblastic/ myofibroblastic spindle cell component, which is often arranged in a storiform array that, when present in the biopsy, helps to establish the differential diagnosis. In those instances in which the diagnosis is not apparent on routine light microscopy, immunohistochemical and/or electron microscopy studies can be very helpful as pleomorphic malignant fibrous histiocytomas do not express mesothelial markers, such as calretinin, WT1, podoplanin or mesothelin, and do not present ultrastructural features indicating mesothelial differentiation.

On occasion, sarcomatoid mesotheliomas can be very anaplastic and may contain bizarre multinucleated tumor cells, which results in a picture that most closely resembles a pleomorphic malignant fibrous histiocytoma; however, these tumors need to be distinguished from the pleomorphic variant of epithelioid mesothelioma. That all of the pleomorphic mesotheliomas in the present series were characterized by marked cellular discohesion, high mitotic activity and prominent epithelioid morphology, including the presence in some cases of poorly formed tubular or papillary structures (Figures 1a and $3 \mathrm{~h}$ ) or deciduoid features (Figure 3a), supports the current belief that these tumors should be regarded as a high-grade variant of epithelioid mesothelioma, ${ }^{19}$ and should not be reclassified as sarcomatoid mesotheliomas as has recently been proposed by some authors. ${ }^{9}$ Further support for this position is also provided by the electron microscopic finding, in the present investigation, of a large number of long slender microvilli that were frequently seen on the cell membrane of the neoplastic cells. This is in contrast to sarcomatoid mesotheliomas in which the cell membranes are usually devoid of microvilli, and if such structures are present, they are sparse and rudimentary. Additionally, the fact that the cytologic examination of the fluid from the pleural effusion in the case in which this material was available for review revealed a large number of neoplastic cells exhibiting features of pleomorphic mesothelioma is another indication that pleomorphic mesotheliomas are better classified as high-grade epithelioid mesotheliomas, rather than as sarcomatoid mesotheliomas, 

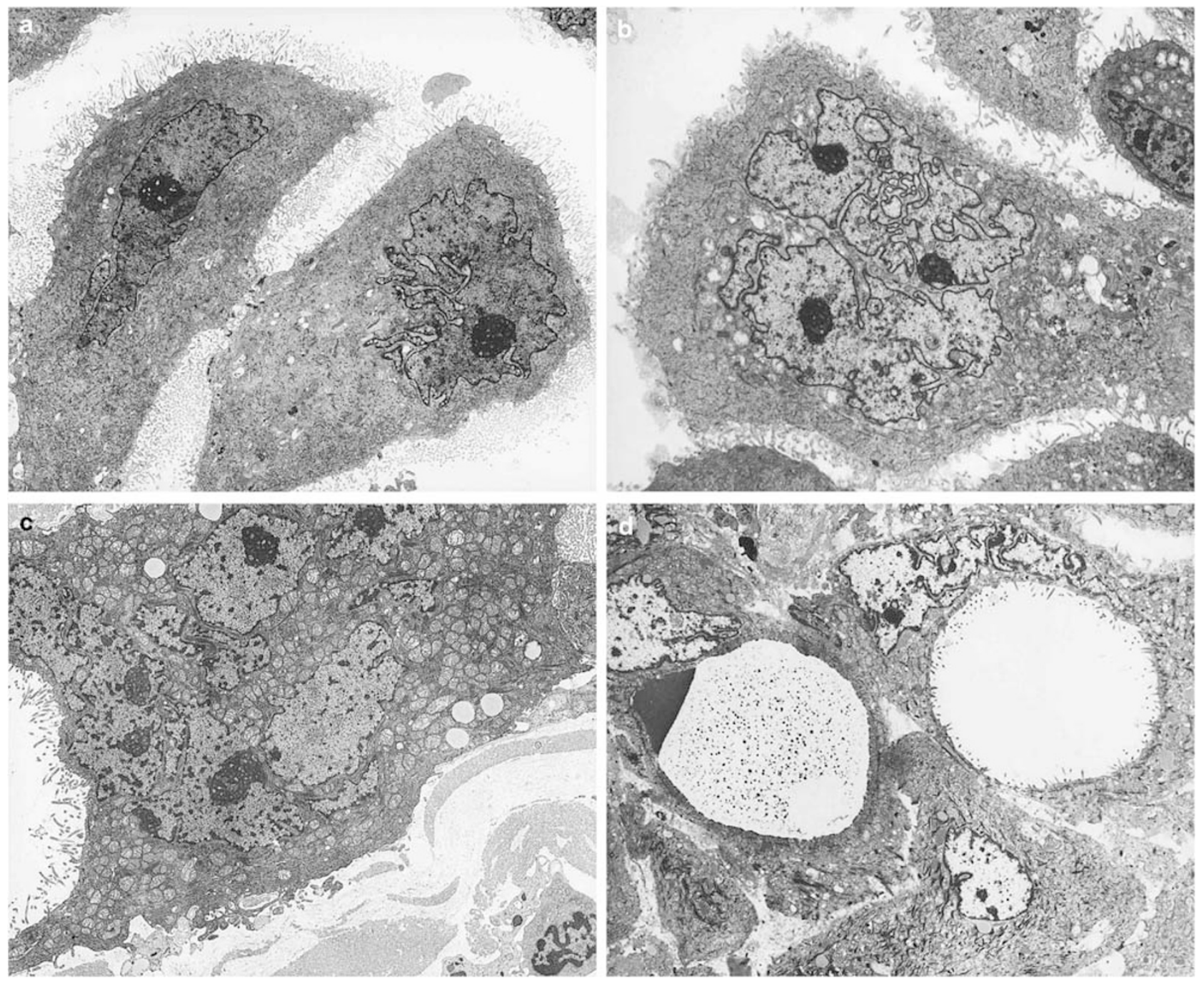

Figure 5 Case 4: (a) Electron micrograph showing two tumor cells that remain barely attached by a few small, poorly developed cell junctions. The cytoplasm is abundant and the nuclei are markedly irregular and each contains a large nucleolus. The cell membranes are covered by numerous, slender microvilli. (b) Binucleated tumor cell showing markedly irregular nuclear contours. One nucleus has two large nucleoli. The apical and lateral portions of the cell membrane are covered by a large number of microvilli. (c) Multinucleated giant tumor cell. The nuclei are irregular and some contain more than one nucleolus. The cytoplasm is abundant and shows a relatively large number of mitochondria. The cell membrane appears to be covered by microvilli. (d) Two of the tumor cells have a large intracytoplasmic lumen. The one on the left contains a small amount of electron dense material, which probably represent proteoglycans. The cell membrane that borders the intra-cytoplasmic lumen of the cell on the right is covered by short microvilli. The cytoplasm of the cell in the lower part of the figure contains abundant bundles of intermediate filaments, which most probably represent keratin $(\mathbf{a}, \times 6000$; $\mathbf{b}$, $\times 14000 ; \mathbf{c}, \times 5500 ; \mathbf{d}, \times 6000)$.

as the latter tumors usually do not exfoliate cells into the effusion fluid. ${ }^{19}$

As Kadota et al ${ }^{9}$ found that the survival of patients with pleomorphic mesothelioma was very similar to that of patients with sarcomatoid mesothelioma, these authors suggested that the pleomorphic subtype of mesothelioma, which is currently classified as an epithelioid mesothelioma according to the 2004 WHO Classification, ${ }^{1}$ should actually be regarded as having a sarcomatoid pattern and these tumors be reclassified as biphasic or sarcomatoid diffuse malignant mesotheliomas. Based on the results of the present investigation, however, the histological and ultrastructural features of mesotheliomas with pleomorphic features more closely resemble those of a conventional epithelioid, rather than a sarcomatoid, mesothelioma, thus justifying that these tumors should continue to be regarded as a histologic subtype of epithelioid mesothelioma.

That all of the patients who underwent extrapleural pneumonectomy in this study were found to have multiple lymph node metastases at the time of surgery is in agreement with the recent report indicating that diffuse epithelioid pleural mesotheliomas exhibiting pleomorphic features behave in a very aggressive manner. Galateau-Salle et $a 1^{17}$ reported in an abstract that the median survival of 44 patients with diffuse epithelioid 

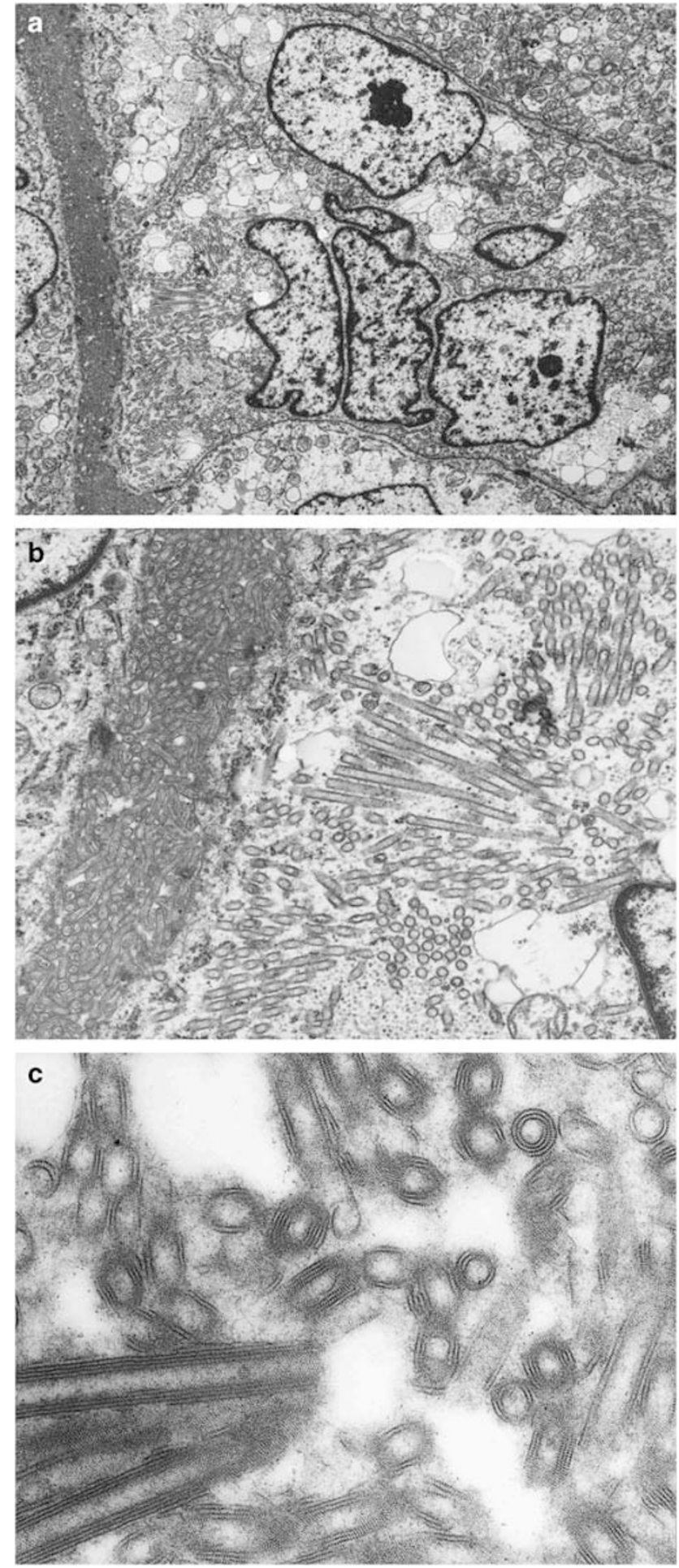

Figure 6 Case 6: (a) Multinucleated giant cell showing large collections of intracytoplamic, tubular crystalloids which are located on the right and left sides of the cells. A large number of long microvilli cover the apical portion of the cell membrane (left). (b) Higher magnification showing one of the clusters of tubular crystalloids and the presence of large, long microvilli on the apical surface of the cell. (c) A very high magnification of the tubular crystalloids within the endoplasmic reticulum. A honeycomb pattern is seen in those which appear longitudinally oriented. The crystalloids are limited by a single or multiple layers of pleated membranous structures, often resulting in a target-like configuration $(\mathbf{a}, \times 7500 ; \mathbf{b} \times 22800 ; \mathbf{c}, \times 75000)$. pleural mesotheliomas with pleomorphic features was 7 months, compared with 13 months for those with more conventional epithelioid mesothelioma. Similar results were obtained in a more recent investigation by Kadota $e t a l^{9}$ who found that among the 232 patients with diffuse pleural mesothelioma that they investigated, those whose tumor exhibited pleomorphic features in over $10 \%$ of the tumor had a worse prognosis. The median survival of the 34 patients who were diagnosed with pleomorphic mesothelioma was 8.1 months, compared with a median survival of 16.2 months for all of the patients who were diagnosed with diffuse epithelioid pleural mesothelioma. The results of these two studies are in concordance with the median survival of 7.3 months obtained in this study.

In conclusion, mesotheliomas with pleomorphic features are characterized by being composed of large, often discohesive, epithelioid cells that vary in size and shape, have abundant dense eosinophilic cytoplasm, and a single or multiple irregular nuclei often containing one or several large nucleoli. The immunohistochemical profile is similar to that seen in other subtypes of epithelioid mesothelioma and electron microscopy often demonstrates that the cell membranes are covered by abundant long, slender microvilli, a feature that is typically present in epithelioid mesotheliomas. Based on these findings, immunohistochemical and electron microscopy studies can be very helpful, not only in assisting in distinguishing these tumors from other malignancies with which they may be confused, but also in providing support for the view that pleomorphic mesotheliomas should continue to be considered a high-grade subtype of epithelioid mesothelioma, not a sarcomatoid mesothelioma as has recently been suggested. As mesotheliomas with pleomorphic features are associated with highly aggressive clinical and biologic behavior, when this subtype of epithelioid mesothelioma is present, it should be mentioned in the pathology report as this can significantly affect the prognosis and patient treatment.

\section{Disclosure/conflict of interest}

The author declares no conflict of interest.

\section{References}

1 Churg A, Roggli V, Galateau-Salle F, et al. Mesothelioma. In: Travis WD, Brambilla E, Müller-Hermelink HK, Harris CC (eds). World Health Organization Classification of Tumours. Pathology \& Genetics. Tumours of the Lung, Pleura, Thymus and Heart. IARC Press: Lyon, France, 2004, pp 128-140.

2 Nascimento AG, Keeney GL, Fletcher CD. Deciduoid peritoneal mesothelioma. An unusual phenotype affecting young females. Am J Surg Pathol 1994;18: 439-445. 
3 Ordóñez NG. Epithelial mesothelioma with deciduoid features: report of four cases. Am J Surg Pathol 2000; 24:816-823.

4 Shia J, Erlandson RA, Klimstra DS. Deciduoid mesothelioma: a report of 5 cases and literature review. Ultrastruct Pathol 2002;26:355-363.

5 Ordóñez NG, Myhre M, Mackay B. Clear cell mesothelioma. Ultrastruct Pathol 1996;20:331-336.

6 Ordóñez NG. Mesothelioma with clear cell features: an ultrastructural and immunohistochemical study of 20 cases. Hum Pathol 2005;36:465-473.

7 Allen TC. Recognition of histopathologic patterns of diffuse malignant mesothelioma in differential diagnosis of pleural biopsies. Arch Pathol Lab Med 2005;129:1415-1420.

8 Itami H, Ohbayashi C, Sakai Y, et al. Pleural malignant mesothelioma with osteoclast-like giant cells. Pathol Int 2010;60:217-221.

9 Kadota K, Suzuki K, Sima CS, et al. Pleomorphic epithelioid diffuse malignant pleural mesothelioma: a clinicopathological review and conceptual proposal to reclassify as biphasic or sarcomatoid mesothelioma. J Thorac Oncol 2011;6:896-904.

10 Mayall FG, Gibbs AR. The histology and immunohistochemistry of small cell mesothelioma. Histopathology 1992;20:47-51.

11 Ordóñez NG. Mesotheliomas with small cell features: report of eight cases. Mod Pathol 2012; advance online publication, 6 January 2012 (e-pub ahead of print).

12 Ordóñez NG. Mesothelioma with rhabdoid features: an ultrastructural and immunohistochemical study of 10 cases. Mod Pathol 2006;19:373-383.

13 Martínez-Consuegra N, Muñoz-Juárez M, Ortiz-Hidalgo C. Unusual multifocal glomeruloid pattern in a well-differentiated papillary mesothelioma of the peritoneum. Int J Surg Pathol 2008;16:426-427.

14 Cook DS, Attanoos RL, Jalloh SS, et al. 'Mucin-positive' epithelial mesothelioma of the peritoneum: an unusual diagnostic pitfall. Histopathology 2000;37:33-36.

15 Rekhi B, Pathuthara S, Ajit D, et al. 'Signet-ring' cells-a caveat in the diagnosis of a diffuse peritoneal mesothelioma occurring in a lady presenting with recurrent ascites: an unusual case report. Diagn Cytopathol 2010;38:435-439.

16 Ordóñez NG. Mesotheliomas with crystalloid structures: report of nine cases, including one with oncocytoid features. Modern Pathol 2012;25:272-281.

17 Galateau-Salle F, Le Stang N, Astoul P, et al. Malignant mesothelioma of the pleura with pleomorphic features: A series of 44 cases. Mod Pathol 2010;23 (Suppl):402A.

18 Miller K, Auld J, Jessup E, et al. Antigen unmasking in formalin-fixed routinely processed paraffin wax-embedded sections by pressure cooking: a comparison with microwave oven heating and traditional methods. Adv Anat Pathol 1995;2:60-64.

19 Churg A, Cagle PT, Roggli VL. Tumors of the Serosal Membranes. AFIP Atlas of Tumor Pathology, Series IV, Fascicle 3. ARP Press: Silver Springs, MD, 2006, p 50.

20 Henderson DW, Shilkin KB, Whitaker D, et al. The pathology of malignant mesothelioma, including immunohistology and ultrastructure. In: Henderson DW, Shilkin KG, Langlois SLD, Whitaker D (eds). Malignant Mesothelioma. Hemisphere: New York, 1992, pp 69-139.

21 Hammar SP, Bockus DE, Remington FL, et al. Mucinpositive epithelial mesotheliomas: a histochemical, immunohistochemical, and ultrastructural comparison with mucin-producing pulmonary adenocarcinomas. Ultrastruct Pathol 1996;20:293-325.

22 Rossi G, Cavazza A, Sturm N, et al. Pulmonary carcinomas with pleomorphic, sarcomatoid, or sarcomatous elements: a clinicopathologic and immunohistochemical study of 75 cases. Am J Surg Pathol 2003;27:311-324.

23 Nonaka D, Tang Y, Chiriboga L, et al. Diagnostic utility of thyroid transcription factors Pax8 and TTF-2 (FoxE1) in thyroid epithelial neoplasms. Mod Pathol 2008;21:192-200.

24 Bishop JA, Sharma R, Westra WH. Pax8 immunostaining of anaplastic thyroid carcinoma: a reliable means of discerning thyroid origin for undifferentiated thyroid tumors of the head and neck. Hum Pathol 2011;42:1873-1877.

25 Laury AR, Hornick JL, Perets R, et al. PAX8 reliably distinguishes ovarian serous tumors from malignant mesothelioma. Am J Surg Pathol 2010;34:627-635.

26 Miettinen M, Franssila KO. Variable expression of keratins and nearly uniform lack of thyroid transcription factor 1 in thyroid anaplastic carcinoma. Hum Pathol 2000;31:1139-1145.

27 Terada T, Nakanuma Y, Matsubara T, et al. An autopsy case of primary angiosarcoma of the pericardium mimicking malignant mesothelioma. Acta Pathol Jpn 1988;38:1345-1351.

28 Lin BT, Colby T, Gown AM, et al. Malignant vascular tumors of the serous membranes mimicking mesothelioma. A report of 14 cases. Am J Surg Pathol 1996;20: 1431-1439.

29 Falconieri G, Bussani R, Mirra M, et al. Pseudomesotheliomatous angiosarcoma: a pleuropulmonary lesion simulating malignant pleural mesothelioma. Histopathology 1997;30:419-424.

30 Del Frate C, Mortele K, Zanardi R, et al. Pseudomesotheliomatous angiosarcoma of the chest wall and pleura. J Thorac Imaging 2003;18:200-203.

31 Dainese E, Pozzi B, Milani M, et al. Primary pleural epithelioid angiosarcoma. A case report and review of the literature. Pathol Res Pract 2010;206:415-419. 